\title{
LA INCORPORACIÓN DEL CONCEPTO DEL BLOQUE DE CONSTITUCIONALIDAD EN MATERIA DE DERECHOS HUMANOS EN MÉXICO.
}

\author{
Alejandra Virginia Morales Morales* \\ Jean Cadet Odimba On'Etambalako Wetshokonda**
}

Fecha de recepción: 24 de noviembre de 2010

Fecha de Aprobación: 13 de abril de 2011

Articulo de Reflexión

\begin{abstract}
Resumen
La Constitución es entendida cómo el eje regulador de un Estado de derecho, cuya finalidad será garantizar a todas las personas por igual sus derechos. Sin embargo, también deben de existir los medios idóneos para garantizar la defensa de la Constitución para conservar la normativa; prevenir su violación, y conservar el orden jurídico.

Para la efectiva protección de los derechos humanos y garantias individuales algunos países de Latinoamérica han incluido en sus constituciones un bloque constitucional, entendido éste como aquellas normas y principios que, sin aparecer formalmente en el articulado del texto constitucional, son utilizados como parámetros del control de constitucionalidad.
\end{abstract}

\section{Palabras clave}

Defensa de la Constitución, Bloque de Constitucionalidad, Derechos Humanos

\section{INCORPORATING THE CONSTITUTIONAL CONCEPT OF BLOCK ON HUMAN RIGHTS IN MEXICO}

\begin{abstract}
Constitution is under tool as the principal regulator of a state of laws which purpose is to ensure to all person equal rights. However it also must exist suitable resources to guarantee the defense of the constitution in order to keep the rules, to prevent its violation and to maintain law and order. For an effective protection of human rights and individual guarantees some American Countries have included in their constitution a constitutional block including such rules and principles that are used as judicial control parameters although these don't appear formally in the constitution al articles.
\end{abstract}

\section{Key words}

Defense of Constitution, Block Constitutionality, Human Rights.

\footnotetext{
Candidata a Maestra en derecho por el posgrado de la Facultad de derecho y Ciencias Sociales de la Universidad Michoacana de san Nicolás de Hidalgo. Correo electrónico: virgyale@live.com.mx

** Licenciatura y Maestría en Derecho de la Universidad de Atenas. Estudios complementarios en Administración Pública Internacional en la Univeridad de Lovaina la Nueva en Belgica. Doctor en Derecho con Mención Honorífica, por la División de Estudios de Posgrado en Derecho de la Facultad de Derecho de la Universidad Nacional Autónoma de México. Estancia posdoctoral en la Maestría de Derecho del Centro Universitario de Ciencias Sociales y Humanidades de la Universidad de Guadalajara. Coordinador General del Centro de Investigaciones Juridicas y Sociales de la Facultad de Derecho y Ciencias Sociales de la Universidad Michoacana de San Nicolás de Hidalgo. Docente-Investigador. jcodimba2002@yahoo.com.mx
} 


\title{
A INCORPORAÇÃO DO CONCEITO DO BLOCO DE CONSTITUCIONALIDADE EM MATÉRIA DE DIREITOS HUMANOS NO MÉXICO
}

\begin{abstract}
Resumo
A Constituição é entendida como a essência reguladora de um Estado de direito, cuja finalidade será garantir a todas as pessoas seus direitos por igual. No entanto, também devem existir os meios idôneos para garantir a defesa da Constituição para conservação da normatividade, prevenir sua violação e manter a ordem jurídica.

Para a efetiva proteção dos direitos humanos e garantias individuais, alguns países da America Latina incluíram em suas constituições um bloco constitucional, entendido como sendo aquelas normas e princípios que, sem aparecer formalmente no artigo do texto constitucional, são utilizados como parâmetros do controle de constitucionalidade.

\section{Palavras-chave}

Defesa da constituição, Bloco de Constitucionalidade, Direitos humanos.
\end{abstract}

\section{LA CONSTITUCIÓN}

Toda sociedad está sujeta a un ordenamiento jurídico con una multiplicidad de normas jurídicas, o lo que es lo mismo en un Estado de Derecho. De acuerdo a lo anterior, se desprende el interrogante de cuáles son las funciones principales del derecho dentro de la sociedad, existen varias pero la principal es la resolución de conflictos.

Para resolver los conflictos es necesaria una regulación jurídica y en este sentido, la principal ley interna es la Constitución Política de cualquier país. Para el constitucionalismo del siglo pasado, el ideal de Constitución era liberal-democrática, la misma Declaración de los Derechos del Hombre y del Ciudadano de 1789 establece en su artículo 16 "Toda sociedad en la cual la garantía de los derechos no está asegurada, ni la separación de los poderes establecida, no tiene Constitución”. (DECLARACIÓN: 1789)

Desde esta conceptualización la Constitución es vista como estructura, política y democrática, la cual regula los órganos del estado fundamental para la sociedad. Doctrinalmente hablando la Constitución puede ser entendida según Ignacio de Otto como: "la expresión de derecho constitucional y cualquier otra en que el término aparezca como adjetivo, se encuentra en su origen ostensiblemente cargada de significado político, evoca de inmediato ideas tales como libertad y democracia, garantía de los derechos de los ciudadanos, limitación del poder". (OTTO: 1999: 11)

La Constitución entendida como el principal sistema de normas jurídicas, como estructura nuclear en una sociedad, puede ser abordada desde una infinidad de puntos de vista, pero siempre se expresa como Birdart Campos señala : "la Constitución de un Estado es la real, verdadera y efectivamente lo ordena, lo hace ser y existir tal cual es, lo compone y lo estructura". (MONROY: 2005: 14 - 42)

Al incorporar el Bloque constitucional en el ordenamiento jurídico mexicano en materia de derechos humanos se estaría hablando de garantizarlos de forma efectiva, puesto que permitirá que aquellos tratados en materia de derechos humanos, ratificados por México, establezcan su jerarquía en la propia Constitución. Así mismo, permite que en la resolución de conflictos no sólo se pueda aplicar lo establecido en la Constitución, sino también otras disposiciones y los principios de valor 
constitucional. Así el Bloque de constitucionalidad es entendido cómo un medio de control de constitucionalidad.

\subsection{La defensa de la constitución}

Héctor Fix Zamudio señala que la defensa de la Constitución está integrada por todos aquellos instrumentos jurídicos y procesales que se han establecido para conservar la normativa constitucional; prevenir su violación; reprimir su desconocimiento; y, lograr el desarrollo y la evolución de las propias disposiciones constitucionales en su doble sentido. Es decir, desde el punto de vista formal, a fin de lograr su paulatina adaptación a los cambios de la realidad político-social y, desde la perspectiva real, su transformación de acuerdo con las normas pragmáticas de la propia carta fundamental.

Todos estos instrumentos que se mencionan como integrantes de la defensa de nuestra Constitución hacen efectivo el Estado de Derecho, para que esto sea más funcional se requiere una Constitución dinámica y más aún con este proceso de cambios y conceptos para tener una armonización y acoplamiento con la realidad que se vive.

En el sistema jurídico mexicano, los instrumentos protectores de la Constitución son:

a) Instrumento Político, que se traduce en la división de poderes;

b) Instrumentos Sociales, que se da con la participación de los grupos sociales y de los partidos políticos;

c) Instrumentos Económicos, que consiste en la regulación de los recursos económicos y financieros (Ley de Ingresos y Presupuesto de Egresos, así como la fiscalización del gasto público); $y$,

d) Técnica Jurídica, la cual tiene sustento en la supremacía constitucional y en el procedimiento dificultado de reforma a la Constitución.

Otro sistema de defensa de la Constitución, mencionadopordoctrinarios, eselquecontempla las denominadas garantías constitucionales, las cuales constituyen los medios jurídicos de naturaleza predominantemente procesal, que se encuentran dirigidos a la reintegración del orden constitucional cuando éste ha sido desconocido o violado por los propios órganos de poder.

Estos instrumentos protectores antes mencionados, no han sido suficientes para lograr el respeto y cumplimiento de las disposiciones constitucionales y más en estos tiempos donde se violentan constantemente los derechos contemplados en la Constitución.

Las garantías constitucionales, también conocidas como medios de control constitucional, tienen el carácter reparador, a diferencia de los instrumentos protectores como los derechos humanos. (BRAVO: 2010: 5) Sin embargo, en la actualidad es necesario que se busquen mecanismos para poder garantizar tanto las garantías individuales como los derechos humanos.

Los países latinoamericanos están avanzando en reconocer en sus constituciones no solamente las garantías individuales, sino también los derechos humanos. Bajo esta perspectiva, sólo en el caso de que un tratado internacional establezca derechos más favorables que aquellos establecidos en la propia Constitución para sus ciudadanos, se puede considerar la supranacionalidad de un tratado sobre la misma Constitución. En este sentido, el tratado internacional de los derechos humanos pasa a formar parte del Bloque constitucional, lo que es equivalente a decir que tienen la misma jerarquía de la Constitución.

Luigi Ferrajoli puntualiza: "Es un hecho que con los procesos actuales de integración jurídica, europea y mundial, el sistema se ha complicado enormemente con el agregado de las fuentes que entran a formar parte del ordenamiento estatal sin siquiera contar con la mediación formal de una ley de ratificación". (FERRAJOLI: 2005: 221-234)

A continuación se mencionarán dos países de Latinoamérica que se toman como ejemplo de 
cómo en los últimos años han incorporado el concepto de Bloque de constitucionalidad en su Constitución

\section{BLOQUE DE CONSTITUCIONALIDAD EN COLOMBIA}

En Colombia, al igual que Argentina, desde hace años han utilizado el Bloque de constitucional en materia de derechos humanos, el cual se hizo mención en la Jurisprudencia de la Corte Constitucional colombiana a partir de 1995. Sin embargo, como concepto se venía aplicando desde años anteriores, utilizando los valores y principios en el texto constitucional para asegurar la permanencia y obligatoriedad del contenido material de la Constitución colombiana. Fajardo Arturo hace mención que:

El Bloque de Constitucionalidad es uno de los más importantes $y$, a la vez, menos claros instrumentos que incluyó la Carta Política de 1991 al sistema normativo colombiano. Se trata de una herramienta de integración del derecho internacional en el orden constitucional colombiano y por ende, de un medio de ampliación de la normatividad constitucional, para el control de la normatividad jerárquicamente inferior $e$, igualmente, para la garantía de los derechos humanos en el país. (FAJARDO: 2007: 2)

Cuando se establece que no es claro, es en virtud de que es imprescindible para los estudiosos del derecho conocer las reglas y subreglas establecidas en la Jurisprudencia por la Corte Constitucional, porque el sólo conocimiento de los textos legales es insuficiente para realizar de manera seria los análisis y argumentos válidos al menos en el ámbito del derecho constitucional. Al respecto la Corte Constitucional colombiana fue precisando de manera paulatina el concepto de Bloque Constitucional para determinar que existen dos sentidos del mismo.

En efecto, la Corte a partir de 1997, empieza a distinguir entre el sentido estricto y el sentido lato del Bloque, para diferenciar entre aquellos instrumentos normativos que se sumaban como fuentes materiales formales al texto de la Constitución y aquellos que se adherían como fuentes de interpretación de las normas constitucionales. (FAJARDO: 2007: 6)

El Bloque de Constitucional colombiano en sentido estricto, corresponde únicamente a las normas de jerarquía constitucional, y Bloque en sentido lato, que incorpora además las otras disposiciones, que sin tener rango constitucional, representan sin embargo un parámetro de constitucionalidad de las leyes. En esta última acepción el Bloque de Constitucionalidad estaría: "conformado no sólo por el articulado de la Constitución, sino entre otros por los tratados internacionales de que trata el artículo 93 de la Carta, por las leyes orgánicas $y$, en algunas ocasiones, por las leyes estatutarias". (VELANDÍA: 2006: 18)

Al respecto Mónica Arango Olaya puntualizó que el Bloque de constitucionalidad colombiano tiene tres niveles diferentes, que la jurisprudencia ha llamado el Bloque en sentido estricto y el Bloque en sentido lato. Estos tres niveles son:

1. Las normas de rango constitucional;

2. Los parámetros de constitucionalidad de las leyes; $y$

3. Las normas que son constitucionalmente relevantes en un caso específico. Por una parte el Bloque de constitucionalidad en sentido estricto está compuesto por:

a. El preámbulo de la Constitución;

b. La Constitución;

c. Los tratados limítrofes de derecho internacional ratificados por Colombia;

d. La ley estatutaria que regula los estados de excepción;

e. Los tratados de Derecho Internacional Humanitario;

f. Los tratados de derecho internacional que reconocen derechos intangibles;

g. Los artículos de los tratados de derecho internacional de derechos humanos ratificados por Colombia, cuando se trate de derechos reconocidos por la Carta;

h. La doctrina elaborada por los tribunales 
internacionales u órganos de control de los tratados de derechos humanos en relación con esas normas internacionales restrictivamente y sólo en determinados casos.

Mientras que por otra parte el Bloque de constitucionalidad en sentido lato como parámetro de constitucionalidad de las leyes está compuesto por:

a. Las leyes orgánicas; y

b. Las leyes estatutarias en lo pertinente. (ARANGO: 2004: 102) Es interesante destacar que la sentencia C-225-95 del Magistrado Ponente: Alejandro Martínez Caballero señala que: El Bloque de constitucionalidad está compuesto por aquellas normas y principios que, sin aparecer formalmente en el articulado del texto constitucional, son utilizados como parámetros del control de constitucionalidad de las leyes, por cuanto han sido normativamente integrados a la Constitución, por diversas vías y por mandato de la propia Constitución. Son pues verdaderos principios y reglas de valor constitucional, esto es, son normas situadas en el nivel constitucional, a pesar de que puedan a veces contener mecanismos de reformas diversas al de las normas del articulado constitucional stricto sensu. (CORTE CONSTITUCIONAL COLOMBIANA: 1995)

En esta sentencia C-225-95 también se establece la prevalencia de tratados de derechos humanos y derechos humanitarios utilizando el Bloque de constitucionalidad al establecer que: el único sentido razonable que se puede conferir a la noción de prevalecía de los tratados de derechos humanos y de derecho internacional humanitario es que éstos forman con el resto del texto constitucional un "Bloque de constitucionalidad", cuyo respeto se impone a la ley. En efecto, de esa manera se armoniza plenamente el principio de supremacía de la Constitución, como norma de normas, con la prevalencia de los tratados ratificados por Colombia, que reconocen los derechos humanos y prohíben su limitación en los estados de excepción. Como es obvio, la imperatividad de las normas humanitarias y su integración en el Bloque de constitucionalidad implica que el Estado colombiano debe adaptar las normas de inferior jerarquía del orden jurídico interno a los contenidos del derecho internacional humanitario, con el fin de potenciar la realización material de tales valores. (CORTE: 1995)

La Corte Constitucional de la República de Colombia se ha ido adecuando al crecimiento de los derechos humanos observados en el ámbito internacional, lo que se ve reflejado en sus jurisprudencias, legitimando el valor de ciertas normas y principios supranacionales que se encuentran incorporados en la Constitución Colombiana y que por lo tanto, son parámetros del control de constitucionalidad, así mismo como parámetros vinculantes de interpretación de los derechos y deberes protegidos por la norma suprema. (ARANGO: 2004: 83) El Bloque Constitucional en Colombia se puede encontrar según la jurisprudencia colombiana en siete artículos: el 9.3 párrafo III, 93, 94, 101, 164 y 214 en su numeral 2 de la Constitución Política Colombiana que a continuación se menciona el contenido de dichos artículos: Artículo 9. Las relaciones exteriores del Estado se fundamentan en la soberanía nacional, en el respeto a la autodeterminación de los pueblos y en el reconocimiento de los principios del derecho internacional aceptados por Colombia. De igual manera, la política exterior de Colombia se orientará hacia la integración latinoamericana y del Caribe. Artículo 53. Párrafo III, Los convenios internacionales del trabajo debidamente ratificados, hacen parte de la legislación interna.Artículo 93. Los tratados y convenios internacionales ratificados por el Congreso, que reconocen los derechos humanos y que prohíben su limitación en los estados de excepción, prevalecen en el orden interno.

Los derechos y deberes consagrados en esta Carta, se interpretarán de conformidad con los tratados internacionales sobre derechos humanos ratificados por Colombia 
Artículo 94. La enunciación de los derechos y garantías contenidos en la Constitución y en los convenios internacionales vigentes, no debe entenderse como negación de otros que, siendo inherentes a la persona humana, no figuren expresamente en ellos.

Artículo 101. Los límites de Colombia son los establecidos en los tratados internacionales aprobados por el Congreso, debidamente ratificados por el Presidente de la República, y los definidos por los laudos arbítrales en que sea parte la Nación.

Los límites señalados en la forma prevista por esta Constitución, sólo podrán modificarse en virtud de tratados aprobados por el Congreso, debidamente ratificados por el Presidente de la República.

Artículo 164. El Congreso dará prioridad al trámite de los proyectos de ley aprobatorios de los tratados sobre derechos humanos que sean sometidos a su consideración por el Gobierno.

Artículo 214, numeral dos: No podrán suspenderse los derechos humanos ni las libertades fundamentales. En todo caso se respetarán las reglas del derecho internacional humanitario. Una ley estatutaria regulará las facultades del Gobierno durante los estados de excepción y establecerá los controles judiciales y las garantías para proteger los derechos, de conformidad con los tratados internacionales. Las medidas que se adopten deberán ser proporcionales a la gravedad de los hechos ${ }^{1}$.

Los doctrinarios colombianos coinciden que el Bloque constitucional sirve para coordinar el derecho interno con las normas internacionalmente acordadas, además no es solamente un avance en la protección de los derechos fundamentales, sino que es una herramienta que garantiza la integración del derecho internacional

Véase la Constitución Política de la República de Colombia actualizada hasta el Decreto 2576 del 27 de julio de 2005. en el orden jurídico colombiano de manera eficiente. El doctrinario Ramelli Arteaga menciona que: "El Bloque de Constitucionalidad surgió por la necesidad de armonizar el contenido del principio de supremacía constitucional con el de primacía del derecho internacional". (SIERRA: 2008: 189 - 207)

La existencia del Bloque Constitucional en Colombia adquiere una gran importancia en materia de derechos humanos, puesto que se podría decir que la Constitución tiene fuerza normativa. Si se partedel Bloque constitucional en susentido amplio, no solamente las normas constitucionales se toman en cuenta para garantizar la protección de un derecho humano, sino que además se pueden tomar en cuenta otras disposiciones y principios para de forma eficaz hacer cumplir dicha garantía.

\section{ARGENTINA Y EL BLOQUE DE CONSTITUCIONALIDAD EN MATERIA DE DERECHOS HUMANOS}

La República de Argentina tenía conflictos respecto de la jerarquía de los tratados internacionales, los cuales fueron resueltos con la reforma constitucional del año 1994. En tal virtud ha ratificado varios instrumentos internacionales de derechos humanos y ha sostenido que los tratados forman parte de su ordenamiento jurídico nacional. Con respecto a la aplicación de los tratados internacionales sobre derechos humanos la república de Argentina no requiere normas reglamentarias internas para ser invocadas $y$ ejercidas en plenitud, es decir, está bajo el sistema de incorporación basado en la teoría monista o también denominado automático a partir de esta reforma constitucional.

Laactual Constitución de Argentina en el artículo31 establece:esta Constitución, las leyes de la Nación que en su consecuencia se dicten por el Congreso y los tratados con las potencias extranjeras son la ley suprema de la Nación; y las autoridades de cada provincia están obligadas a conformarse a ella, no obstante cualquiera disposición en contrario que 
contengan las leyes o Constituciones provinciales, salvo para la Provincia de Buenos Aires, los tratados ratificados después del pacto del 11 de noviembre de 1859. En este supuesto equipara a los tratados internacionales con las leyes que dicta el Congreso, por lo tanto, se podría decir que la jerarquía de los instrumentos internacionales de derechos humanos en Argentina está en una jerarquía Constitucional. Según lo establecido en la propia Constitución, la cual en su artículo 75, fracción 22, señala: Corresponde al Congreso:...22. Aprobar o desechar tratados concluidos con las demás naciones y con las organizaciones internacionales y los concordatos con la Santa Sede. Los tratados y concordatos tienen jerarquía superior a las leyes. La Declaración Americana de los Derechos y Deberes del Hombre; la Declaración Universal de los Derechos Humanos; la Convención Americana de Derechos Humanos; el Pacto Internacional de Derechos Económicos, Sociales y Culturales; el Pacto Internacional de Derechos Civiles y Políticos y su Protocolo Facultativo; la Convención sobre la Prevención y la Sanción del Delito de Genocidio, la Convención Internacional sobre la Eliminación de todas las Formas de Discriminación Racial; la Convención sobre la Eliminación de todas las Formas de Discriminación contra la Mujer; la Convención contra la Tortura y otros Tratos o Penas Crueles, Inhumanos o Degradantes; la Convención sobre los Derechos del Niño; en las condiciones de su vigencia, tienen jerarquía constitucional, no derogan artículo alguno de la primera parte de esta Constitución y deben entenderse complementarias de los derechos y garantías por ella reconocidos. Sólo podrán ser denunciados en su caso, por el Poder Ejecutivo Nacional, previa aprobación de las dos terceras partes de la totalidad de los miembros de cada cámara.

Este artículo señala que todo tratado celebrado con potencias extranjeras por el poder Ejecutivo, requiere para su validez la aprobación del congreso para convertirse en ley. Además, todos estos tratados o convenciones de carácter internacional deben ser celebrados con sujeción a los principios de derecho público que caracterizan la forma republicana de gobierno, tal como ella resulta adaptada al país de Argentina por la Constitución en su conjunto armónico de sus disposiciones correlacionadas entre sî". (CONSTENLA: 2003: 114) Algunos autores señalan que con las reformas de la constitución de la República de Argentina 1994, se estableció un Bloque constitucional entendido este, como: aquellas normas y principios que sin aparecer formalmente en los artículos que conforman el texto constitucional son utilizados como parámetros del control de constitucionalidad de las leyes, por lo que han sido normativamente integrados a la Constitución, por diversas vías. (ARANGO: 2004: 1)

De acuerdo a lo establecido en el artículo 75 inciso 22, los aspectos relevantes de este Bloque constitucional es porque:

a) Establece la jerarquía supralegal de los tratados internacionales celebrados por este país con otros organismos internacionales o estados

b) Le otorga el rango constitucional a las declaraciones, convenciones y algunos tratados en materia de derechos humanos, es decir, a un grupo de instrumentos internacionales en materia de derechos humanos los ponen en un plano de igualdad con la Constitución de la República de Argentina

c) Además establecen los mecanismos de incorporación de los demás tratados y convenciones que se celebren o ratifiquen en un futuro, en materia de derechos humanos

d) Así como también estableció un proceso legislativo particular para incorporar otros tratados de derechos humanos.

De lo anterior, se debe resaltar que "los nuevos parámetros de control, integran lo que se le denomina como nueva fórmula de validez del derecho positivo argentino y son agrupados por la doctrina bajo la noción de Bloque de constitucionalidad federal"; (ARANGO: 2004:

1) por lo tanto, una ley es válida sólo si no contradice el Bloque Constitucional. 
Con las reformas estructurales de 1994 realizadas a la Constitución de Argentina, hay autores que sostienen que se transformó la clásica pirámide Kelseniana en un especie de trapecio al truncar o achatar, el triángulo superior. El reinado de la Constitución dejó de ser absoluto y exclusivo para constituirse en un gobierno mancomunado junto a tratados internacionales de derechos humanos que pasaban a tener su misma jerarquía. (PIZZOLO: 2002: 514)

\section{BLOQUE CONSTITUCIONAL EN MÉXICO PARA INCORPORAR INSTRUMENTOS EN MATERIA DE DERECHOS HUMANOS}

En el caso de México, el primer antecedente que se tiene de esta figura de Bloque constitucional, está en una tesis de jurisprudencia que pronunció la Suprema Corte de Justicia en materia electoral. (JURISPRUDENCIA: 2007: 1641) Sin embargo, no ha cobrado fuerza el concepto de Bloque de constitucionalidad en ninguna materia, incluyendo la materia de derechos humanos.

En México en los últimos años, los derechos humanos, han retomado fuerza su observancia, protección, promoción, estudio y divulgación. Esta situación ha permitido que se tengan grandes avances en materia de derechos humanos, aunque la cultura de protección y promoción de los derechos humanos no es tan marcada como sucede en países como Argentina y Colombia, que han integrado el Bloque de constitucionalidad en materia de derechos humanos en el articulado de la propia Constitución. Otros países como Francia y España también han integrado de manera clara y precisa el Bloque de Constitucionalidad como control de constitucionalidad, entendiendo éste como el mecanismo jurídico mediante el cual se asegura cabalmente el cumplimiento de las normas constitucionales dentro de un ordenamiento jurídico.

En relación al Bloque Constitucional, José de Jesús Muñoz Navarro manifiesta que: el Bloque de Constitucionalidad implica incorporar un parámetro de control constitucional, con normas y principios que no están contemplados directa o formalmente en la Constitución, pero que de conformidad con la propia Carta Magna, deben ser considerados con grado constitucional para el control de actos y leyes. El concepto entonces cobra trascendencia, para ampliar la esfera de derechos a ser protegidos por nuestros tribunales, a través de los diversos medios de control constitucional, específicamente, los controles jurisdiccionales de la Constitución. (MUÑOZ: 12)

En la Constitución Política de los Estados Unidos Mexicanos en su artículo 133, establece una pauta mínima, sólo señala, "las leyes del Congreso de la Unión que emanen de ella y todos los Tratados que estén de acuerdo con la misma, serán la Ley Suprema de toda la Unión". El texto constitucional hace mención a unos tratados, no obstante, queda sin esclarecer si se trata de tratados internacionales en materia de derechos humanos; tampoco señala la jerarquía que deben tener dichos tratados en su ordenamiento jurídico interno. Para lograr una efectiva protección de los derechos humanos es imprescindible que se haga referencia a la jerarquía que guardan los tratados de derechos humanos en un ordenamiento jurídico de manera clara y sencilla. Por ejemplo, Francia hace su clasificación en leyes básicas y ordinarias, además señala un tercer tipo de leyes, que son aquellas normas sujetas a control constitucional que no están establecidas en las leyes básicas ni ordinarias.

En este contexto, Muñoz Navarro dice que el término ya fue incorporado a nuestro sistema jurídico mexicano por la Jurisprudencia de la Corte-en materia electoral-y, por lo tanto puede ser invocado para integrar preceptos, principios $y$ valores que se consideren incorporados a la Constitución por referencia o señalamiento de la propia Constitución en materia de control de la constitucionalidad; eso dependerá solamente de la interpretación de nuestros tribunales federales. No obstante, no se avanzaría porque esta acción implicaría llenar las lagunas de ley por medio de la jurisprudencia de la Corte 
que la Constitución no cubre. Además se debe recordar que el sistema jurídico mexicano se basa en leyes escritas y no por precedente, por lo que las jurisprudencias de la Corte no se toman como elementos trascedentes cuando se trata de Bloque de constitucionalidad.

Por lo tanto, en el estado jurídico mexicano, la pregunta obligada sería ¿Cómo integrar un Bloque constitucional en materia de derechos humanos?, considerando que el artículo 133 constitucional no ofrece suficientes elementos para garantizar la protección de los derechos humanos y que las jurisprudencias no aseguran la promoción y protección de los mismos

Con la finalidad de dar respuesta al anterior cuestionamiento y a raíz de la creciente necesidad de brindar las condiciones para una buena promoción y protección de los derechos humanos, se realizaron esfuerzos para conciliar una reforma constitucional en materia de derechos humanos. En este contexto, el pasado 8 de Marzo del 2011 se aprobó un proyecto de decreto, donde se modifican varios artículos de la Constitución Política de los Estados Unidos Mexicanos, los cuales son: 1, 3, 11, 15, 18, 29, 33, 89, 97, 102 y 105 a esta reforma se le denominó reforma constitucional en materia de derechos humanos. Dentro de los avances en la materia en cuestión sobresalen varios aspectos, como la incorporación del término de derechos humanos en la Constitución, se determinó la inclusión de los derechos humanos en la educación y en la política exterior; la prohibición de celebrar tratados internacionales que vulneren los principios de la universalidad, interdependencia, indivisivilidad y progresividad de los derechos, se reconoció el principio pro-persona, se le otorgó más atribuciones a la Comisión Nacional de derechos Humanos, entre otros aspectos. Cabe hacer mención que el Senado mexicano remitió la minuta de reforma para su aprobación por parte de las legislaturas estatales, tal y como lo ordena la Constitución política de los Estados Unidos Mexicanos. Sólo falta esperar que aprueben las legislaturas. Y una vez concretado esta reforma sea entonces una realidad formal.
Hay doctrinarios que dicen que con esta reforma se puede hablar ya de un Bloque de Constitucionalidad en materia de derechos humanos, dado que el artículo $1^{\circ}$ quedaría de la siguiente manera:

"En los Estados Unidos Mexicanos, todas las personas gozarán de los derechos humanos reconocidos en esta Constitución y en los tratados internacionales de los que el Estado mexicano sea parte, así como de las garantías para su protección, cuyo ejercicio no podrá restringirse ni suspenderse, salvo en los casos y bajo las condiciones que esta Constitución establece.

Las normas relativas a los derechos humanos se interpretarán de conformidad con esta Constitución y con los tratados internacionales de la materia favoreciendo en todo tiempo a las personas la protección más amplia.

Todas las autoridades, en el ámbito de sus competencias, tienen la obligación de promover, respetar, proteger y garantizar los derechos humanos de conformidad con los principios de universalidad, interdependencia, indivisibilidad y progresividad. En consecuencia, el Estado deberá prevenir, investigar, sancionar y reparar las violaciones de los derechos humanos, en los términos que establezca la ley".

La reforma es un gran paso hacia una adecuada protección de los derechos humanos, puesto que se vislumbra la implementación de un Bloque de Constitucionalidad, dado que un elemento de esta figura es la aparición de las normas de derechos humanos sin que estén expresamente en la Constitución. No obstante, aún carece de otros elementos que son básicos dentro del concepto de Bloque de constitucionalidad.

Los elementos considerados en el concepto de Bloque de constitucionalidad de Argentina son: 
1. Normas o tratados en materia de derechos humanos que aparecen expresamente en la Constitución

2. Les otorgan un rango Constitucional

3. Existe una sistematización de leyes

4. Remisiones de los tratados de derechos humanos en la legislación interna

5. Los tratados tienen igual fuerza constitucional

Mientras que el concepto de Bloque de constitucionalidad en el caso de Colombia integra elementos como:

1. Las normas o tratados en materia de derechos humanos aparecen expresamente en la Constitución

2. Las normas o tratados tienen rango constitucional

3. Las normas o tratados se remiten al sistema jurídico interno.

Al realizar el análisis de los elementos en relación al concepto de Bloque de constitucionalidad se puede observar la coincidencia entre los dos sistemas jurídicos en cuanto a tres puntos claves: aparición de los tratados de derechos humanos en la Constitución, que tienen estos tratados rango constitucional y que se remiten a los tratados en el sistema jurídico interno. Por lo tanto, para asegurar la formación de un Bloque de constitucionalidad en materia de derechos humanos, a partir de la reciente reforma, se tendrían que cumplir con tales elementos. En primer lugar, se puede observar que se cumple con el primer elemento, que es la aparición no expresa de los tratados de derechos humanos: "todas las personas gozarán de los derechos humanos reconocidos en esta Constitución y en los tratados internacionales de los que el Estado mexicano sea parte". Otro elemento es la jerarquía que guardan los tratados de derechos humanos en relación a la legislación en su conjunto y se puede observar que en el nuevo texto del artículo $1^{\circ}$, no menciona nada respecto de la jerarquía que deben de tener los tratados en materia de derechos humanos, ya que sólo se limita a incorporar el concepto de derechos humanos. Al respecto, y de manera comparada la Constitución Argentina declara de forma precisa la jerarquía constitucional para los tratados en materia de derechos humanos en su artículo 75 inciso 22. Otro ejemplo de la claridad en relación a la jerarquía es la Constitución Colombiana, la cual en su artículo 93 establece que los tratados en materia de derechos humanos prevalecen en el orden interno.

La remisión es otra característica del Bloque de constitucionalidad y en el caso de la Constitución de Argentina claramente se define al establecer los tratados de acuerdo a su importancia y reconocerles igual fuerza constitucional. El elemento de remisión no se expresa en el texto del nuevo artículo $1^{\circ}$. Por lo tanto, desde nuestro punto de vista no se puede hablar de un Bloque de Constitucional en materia de derechos humanos considerando que sólo cumple con un elemento de los tres elementos que de manera general conforman el concepto de Bloque de constitucionalidad.

Independientemente de que no se cumple a cabalidad con los elementos que forman el Bloque de constitucionalidad, cabe hacer el reconocimiento a México que pretende siempre avanzar de manera paulatina para lograr que los derechos humanos sean reconocidos y con estas reforma es un claro ejemplo de que tal vez en un corto tiempo se pueda incluir un Bloque de constitucionalidad en materia de derechos humanos en la Constitución Mexicana.

Después de analizar la reforma en materia de derechos humanosen Méxicoyel seguimiento del Bloque de constitucionalidad en diversos países, consideramos que la verdadera incorporación de un Bloque de Constitucionalidad sería al reformar el artículo 133 constitucional, de los tratados internacionales, con la finalidad de que se especifique la jerarquía de las normas que conforman el ordenamiento jurídico nacional, así como los tratados internacionales en materia de derechos humanos. Los derechos humanos por su importancia, están en constante cambio y por lo tanto es necesario adecuar nuestro sistema jurídico mexicano, la reforma antes mencionada 
contribuye a esa necesidad de adecuar el sistema jurídico mexicano con el internacional.

En el caso de México no había existido un avance tan importante como el que ahora se vislumbra con la esperada reforma, en materia de derechos humanos. De ahora en adelante, esperemos que juristas, legisladores y doctrinarios tomen en cuenta el concepto de Bloque de constitucionalidad en materia de derechos humanos y le den la importancia que se merece a esta figura para estar en posibilidades de proteger y garantizar los derechos humanos de sus ciudadanos desde otro nivel.

\section{CONCLUSIONES}

México tiene la obligación de asegurar de manera eficaz, el cumplimiento tanto de las garantías individuales como de los derechos humanos. Por tal razón, es de vital importancia adecuar la Constitución Política de los Estado Unidos Mexicanos y una alternativa sería la incorporación del concepto de Bloque constitucional en materia de derechos humanos, con lo cual se lograría la efectiva aplicación de los derechos humanos contenidos específicamente en los tratados internacionales en esta materia, además se podría armonizar el derecho interno con el derecho internacional de los derechos humanos. El artículo 133 Constitucional, el cual hace referencia a los tratados internacionales, no obstante, la aplicación de tales normas es ineficiente, dado que tales normas no son tomadas en cuenta por los juristas. Con la finalidad implementar mayor fuerza en materia de derechos humanos, se modificó el artículo $1^{\circ}$ Constitucional, pensando en que podía funcionar tal reforma como un Bloque de constitucionalidad. Una vez realizando su análisis, se observa que algunos elementos del Bloque de constitucionalidad, como la jerarquía de los tratados en el derecho interno, no son claramente incorporados en esta reforma.

Por lo tanto, se podría decir que con la minuta de reforma constitucional en materia de derechos humanos, estamos en posibilidades de que en un futuro se pueda hablar de la figura de Bloque de constitucionalidad en materia de derechos humanos. Como propuesta para completar el Bloque de constitucionalidad en materia de derechos humanos se propone adecuar el artículo 133 y establecer en dicho artículo la jerarquía de los tratados internacionales en materia de derechos humanos.

\section{BIBLIOGRAFÍA}

ARANGO, M. (2004). El Bloque de constitucionalidad en la jurisprudencia de la Corte Constitucional de Colombia, En precedente, anuario Jurídico 2004. Cali, Colombia: Centro de Investigaciones Sociojurídicas, Universidad Icesi.

BRAVO, V. M. (2010). Medios de control de constitucionalidad en México y España, Suprema Corte de Justicia de la Nación. Ciudad de México, México.

CONSTENLA, R. C. (2003, julio - diciembre). Aplicación del derecho internacional de los derechos humanos por los tribunales de la República Argentina, Revista del Instituto Interamericano de Derechos Humanos, Justicia y Seguridad. Argentina:

FAJARDO L. A. (2007, julio - diciembre). Contenido y alcance jurisprudencial del Bloque de constitucionalidad en Colombia. Bogotá, Colombia: Universidad Sergio Arboleda.

FERRAJOLI, L. (2005). ¿Democracia sin Estado?. Publicado en Estudios en Homenaje a Don Jorge Fernández Ruiz. Derecho Constitucional y Política, coordinado por David Cienfuegos Salgado y Miguel Alejandro López Olvera. Ciudad de México, México.

JURISPRUDENCIA. (2007). EstatutodeGobierno del Distrito Federal, junto con la Constitución Política de los Estados Unidos Mexicanos, integra Bloque de Constitucionalidad. Tesis 18/2007, Semanario Judicial de la Federación y su Gaceta, 15,1641 . 
MONROY, M. G. Concepto de Constitución. Colombia. www.juridicas.unam.mx.

MONROY, M. G. (2005). Concepto de Constitución, Colombia, Anuario de Derecho Constitucional Latinoamericano. (Tomo I.)., 20051, Ciudad de México, México: Instituto de Investigaciones Jurídicas de la UNAM

MUÑOZ, J. J. El Bloque Constitucional como parámetro del Control Constitucionalidad en México. Revista Debate Social, ITESO Guadalajara, 23, 12.

OTTO, I. (1999). Derecho Constitucional: sistema de fuentes. Barcelona, España: Ariel.

PIZZOLO. (2002). Los mecanismos de protección en el sistema interamericano de derechos humanos y el derecho interno de los países, México, derecho internacional de los derechos humanos, Memoria del VII Congreso Iberoamericano de Derecho Constitucional. UNAM,

(PIZZOLO, 2002, p. 514)
SIERRA, H. (2008). La administración de justicia en el estado social de derecho privatizado. Revista Jurid, 5, 189 - 207.

(SIERRA, 2008, 189 - 207)

VELANDÍA, A. (2006). Prospectiva constitucional del estatuto de Roma. Revista Prolegómenos, Derechos y Valores, IX, 18, 153,173.

(VELANDÍA, 2006, p. 18)

\section{Legislación}

CONSTITUCIÓN POLÍTICA DE LA NACIÓN DE ARGENTINA.

CONSTITUCIÓN POLÍTICA DE LA REPÚBLICA DE COLOMBIA.

CONSTITUCIÓN POLÍTICA DE LOS ESTADOS UNIDOS MEXICANOS.

CORTE CONSTITUCIONAL COLOMBIANA. (1995). M.P. MARTÏNEZ, A. Sentencia, C -225-95.

DECLARACIÓN DE LOS DERECHOS DEL HOMBRE Y DEL CIUDADANO. (1789). 